\title{
Dual task interference while walking in chronic stroke survivors
}

\author{
Joon-Ho Shin ${ }^{a}$, Hyun Choi ${ }^{b}$, Jung Ah Lee ${ }^{b}$, Seon-deok Eun ${ }^{b}$, Dohoon Koo ${ }^{c}$, JaeHo Kim ${ }^{d}$, Sol Lee ${ }^{c}$, \\ KiHun $\mathrm{Cho}^{\mathrm{e}}$
}

${ }^{a}$ Department of Rehabilitation Medicine, National Rehabilitation Center, Seoul, Republic of Korea

${ }^{b}$ Department of Clinical Research for Rehabilitation, National Rehabilitation Research Institute, National Rehabilitation Center, Seoul, Republic of Korea

'Department of Rehabilitation Policy and Standardization, National Rehabilitation Research Institute, National Rehabilitation Center, Seoul, Republic of Korea

${ }^{\mathrm{d} D e p a r t m e n t}$ of Rehabilitative \& Assistive Technology, National Rehabilitation Research Institute, National Rehabilitation Center, Seoul, Republic of Korea

${ }^{\text {e}}$ Department of Physical Therapy, Uiduk University, Gyeongju, Republic of Korea

Objective: Dual-task interference is defined as decrements in performance observed when people attempt to perform two tasks concurrently, such as a verbal task and walking. The purpose of this study was to investigate the changes of gait ability according to the dual task interference in chronic stroke survivors.

Design: Cross-sectional study.

Methods: Ten chronic stroke survivors ( 9 male, 1 female; mean age, 55.30 years; mini mental state examination, 19.60; onset duration, 56.90 months) recruited from the local community participated in this study. Gait ability (velocity, paretic side step, and stride time and length) under the single- and dual-task conditions at a self-selected comfortable walking speed was measured using the motion analysis system. In the dual task conditions, subjects performed three types of cognitive tasks (controlled oral word association test, auditory clock test, and counting backwards) while walking on the track.

Results: For velocity, step and stride length, there was a significant decrease in the dual-task walking condition compared to the single walking condition $(p<0.05)$. In particular, higher reduction of walking ability was observed when applying the counting backward task.

Conclusions: Our results revealed that the addition of cognitive tasks while walking may lead to decrements of gait ability in stroke survivors. In particular, the difficulty level was the highest for the calculating task. We believe that these results provide basic information for improvements in gait ability and may be useful in gait training to prevent falls after a stroke incident.

Key Words: Cognition, Gait, Stroke

\section{Introduction}

Walking is a complex movement that requires movement strategies and executive abilities of the upper central body in a pattern requiring continuous and repetitive coordination of several segments [1]. Cognitive and muscle weakness, asymmetrical postural control, and impaired coordination abilities after stroke have resulted in slow walking cycles and decreased gait speeds, asymmetric assurance and vaulting, and gait patterns with a short stance phase and a long swing phase on the paralyzed side, which makes independent performance of daily activities difficult [2]. Thus, improvement in walking ability is an essential goal for stroke rehabilitation.

Received: 27 August, 2017 Revised: 12 September, 2017 Accepted: 13 September, 2017

Corresponding author: KiHun Cho

Department of Physical Therapy, Uiduk University, 261 Donghae-daero, Gangdong-myeon, Gyeongju 38004, Republic of Korea Tel: 82-54-760-1795 Fax: 82-54-760-1179 E-mail: mamiya34@gmail.com

(c) This is an Open-Access article distributed under the terms of the Creative Commons Attribution Non-Commercial License (http://creativecommons.org/licens es/by-nc/4.0) which permits unrestricted non-commercial use, distribution, and reproduction in any medium, provided the original work is properly cited.

Copyright ( 2017 Korean Academy of Physical Therapy Rehabilitation Science 
Restoration of walking ability is an important factor for improving the quality of life and independence for persons with stroke. However, despite steady training, $30 \%$ of stroke patients are unable to walk without assistive devices, and only $7 \%$ are reported to be able to walk outside [3]. Various therapeutic approaches such as task-oriented training, feedback training, and treadmill training have been used as an attempt to improve walking ability of stroke survivors [4]. However, since this approach is mainly applied to a hospital environment and does not reflect gait ability performed in an actual community environment, there is controversy about the sustainability of its efficiency and its effectiveness in a real outdoor environmental setting. Palta and ShumwayCook [5] have mentioned that in order for people to acquire stability and independence with gait, one must be able to withstand the eight environmental demands, such as the environmental condition, regional characteristics, external factors, concentration requirements, attitude change, level of traffic volume, time restraints, as well distance. In addition, Hunter and Hoffman [6] reported that for the purpose of gait safety and fall prevention, concentration for postural control is required. Therefore, in recent years, various studies have been attempted on dual-task training to investigate for changes in functional movements during simultaneous execution of two tasks. Restraints to performing dual tasks for stroke survivors include having a dependent daily life, decreased community involvement, and major fall risk factors [7]. According to previous studies, the addition of a cognitive task during gait led to a decrease in walking ability in persons with stroke, and walking ability was remarkably reduced with the addition of a task that involved speaking [8]. Hyndman et al. [9] reported that it is possible to produce improvements in postural control ability in stroke survivors through a dual-task training program. Another study reported that dual-task training programs promote increases in cerebral blood flow [10].

In particular, simultaneous performance of standing or gait and a cognitive task, such as calculating or doing word search, are used to investigate the interaction between concentration and exercise capacity [11]. According to previous studies, dual-task performance has been reported to increase postural fluctuation and decrease gait ability in persons with stroke [8], which was reported as a major factor in inhibiting the automatic gait pattern [11]. When persons with stroke attempt to maintain their balance while performing a dual task, the attention capacity is reduced, making it difficult for them achieve postural control [12]. In addition, it has been re- ported that postural control ability may depend on the type of cognitive task performed [13]. However, studies examining changes in walking ability of persons with stroke according to task type and various difficulty levels are insufficient. Therefore, the purpose of this study was to investigate the changes in walking ability according to the type and difficulty levels of the dual-tasks performed in persons with chronic stroke, and to utilize the results for the purpose of developing training programs to improve walking ability of persons with stroke.

\section{Methods}

\section{Participants}

Twelve community dwellers with chronic stroke were recruited for this study based on the following selection criteria: subjects with an onset of stroke at least 6 months ago, have an mini mental state examination (MMSE) score of $<24$, and those with no musculoskeletal diseases affecting gait. Those with unilateral neglect were excluded from the study. Out of the twelve subjects, two were excluded from the study due to participating in other studies, leaving a total of 10 subjects for final analysis ( 9 males, 1 female; mean age, 55.30 years; MMSE, 19.60; duration of onset, 56.90 months). The characteristics of the study subjects are shown in Table 1. All subjects fully understood the purpose of the study and voluntarily provided their informed consent for participation of the study. This study was approved by the Institutional Review Board of the National Rehabilitation Center (IRB No. NRC-2013-01-001).

\section{Procedures}

This study used a cross-sectional, single group, and re-

Table 1. General characteristics of the subjects $(\mathrm{N}=10)$

\begin{tabular}{lc}
\hline \multicolumn{1}{c}{ Parameter } & n or mean (SD) \\
\hline Paretic side & \\
Left/right & $6 / 4$ \\
Sex & \\
Male/female & $9 / 1$ \\
Etiology & \\
Infarction/hemorrhage & $4 / 6$ \\
Age (yr) & $55.30(12.03)$ \\
Height $(\mathrm{cm})$ & $164.58(8.80)$ \\
Weight $(\mathrm{kg})$ & $68.95(8.43)$ \\
Onset duration (mo) & $56.90(77.61)$ \\
Mini mental state examination (score) & $19.60(1.50)$ \\
\hline
\end{tabular}


peated-measures design to investigate changes in walking ability during dual-task performance in persons with chronic stroke.

Subjects were asked to perform three types of cognitive tasks (controlled oral word association test [COWAT], auditory clock test, and counting backwards) while walking on a 5-meter circular track for 1 minute. The single walking task was performed for 1 minute on the 5-meter circular track. Subjects walked at a comfortable speed for each task. The three cognitive tasks and the single gait task were performed randomly and a 30 -second rest period was provided between each task.

The COWAT [14-16] task was performed using the category fluency test where subjects were asked to say the words relevant to a certain category while walking on a specified track for one minute. The verbal contents were recorded by a tape recorder. The categories used for the fluency tests included names of animals, fruit, and food.

For the auditory clock test $[14,17]$ if the hour and minute hands were in the same direction of the time that was given auditorily, subjects were to asked to say 'yes' and if they were in the opposite direction, the subjects were asked to say 'no.' Subjects walked on the specified track for 1 minute.

For the counting backwards task $[18,19]$, subjects were given a three-digit number and were asked to continuously subtract by 7 while walking on the specified track for 1 minute.

Velocity, step and stride length, and step and stride time were evaluated using a 3-dimensional (3D) motion analysis system (Vicon Motion Systems Ltd., Oxford, UK) to assess gait ability. Eight infrared cameras (Vicon T20; Vicon Motion Systems Ltd.) were used for 3D motion analysis, and the operation frequency was set to $120 \mathrm{~Hz}$ (sampling frequency). Before the start of the experiment, the subject's body size was measured and 16 infrared reflective markers were attached to the subject's lower limb according to the Plug-In-Gait lower body model criteria. The 3D motion analysis equipment was calibrated according to the manual of the manufacturer and the experiment was conducted. The Pedar-X system (Novel, Gmbh, Germany) was used to specify the gait event and synchronized with the Vicon System.

\section{Data analysis}

The IBM SPSS Statistics ver. 21.0 (IBM Co., Armonk, NY, USA) was used for statistical analysis of the collected information. The results were expressed as mean and standard deviation. All variables were normally distributed. Oneway repeated measures analysis of variance was used to compare the difference in the effects of dual-task interference between simple walking and dual-task walking, and the least significant difference was used for post-hoc analysis. Statistical significance was set at $p<0.05$.

\section{Resuts}

General characteristics of the subjects are shown in Table 1. Changes of gait ability under the dual task condition are summarized in Table 2. For velocity, step and stride length, there was a significant decrease in dual-task walking ability compared to single walking $(p<0.05$; Figure 1$)$.

\section{Discussion}

Through previous studies on dual-tasks, it was found that in dual tasks, it was confirmed that the degree of improvement of postural control ability was depended on the added cognitive task $[13,20]$. Therefore, it is necessary to compare various types of cognitive tasks that are added when applying dual tasks to persons with stroke, and to select cognitive tasks that can effectively improve independent daily life or

Table 2. Changes of gait ability under the dual task condition

\begin{tabular}{lcccccc}
\hline \multicolumn{1}{c}{ Parameter } & $\begin{array}{c}\text { Single } \\
\text { walking (A) }\end{array}$ & $\begin{array}{c}\text { COWAT } \\
(\mathrm{B})\end{array}$ & $\begin{array}{c}\text { Auditory } \\
\text { clock test (C) }\end{array}$ & $\begin{array}{c}\text { Counting } \\
\text { backward (D) }\end{array}$ & F (p) & $\begin{array}{c}\text { Post-hoc } \\
(\text { LSD })\end{array}$ \\
\hline Velocity (cm/s) & $51.80(16.59)$ & $45.90(14.41)$ & $45.80(13.83)$ & $45.10(14.85)$ & $7.513(0.001)$ & A-B, A-C, A-D \\
P-Stride length (cm) & $75.20(19.43)$ & $72.00(13.18)$ & $69.60(12.89)$ & $68.20(16.11)$ & $2.537(0.078)$ & A-D \\
P-Stride time (sec) & $1.50(0.28)$ & $1.65(0.34)$ & $1.59(0.29)$ & $1.58(0.28)$ & $2.284(0.102)$ & \\
P-Step length (cm) & $40.90(10.57)$ & $37.70(8.59)$ & $37.20(8.08)$ & $36.40(8.63)$ & $5.549(0.004)$ & A-B, A-C, A-D \\
P-Step time (sec) & $0.68(0.12)$ & $0.72(0.13)$ & $0.69(0.10)$ & $0.66(0.12)$ & $2.042(0.132)$ & \\
\hline
\end{tabular}

Values are expressed as mean (SD). Analyzed by one-way repeated measure ANOVA.

COWAT: controlled oral word association test, P: paretic side, A-B: significant differences between single walking and COWAT, A-C: significant differences between single walking and auditory clock test, A-D: significant differences between single walking and counting backward. 


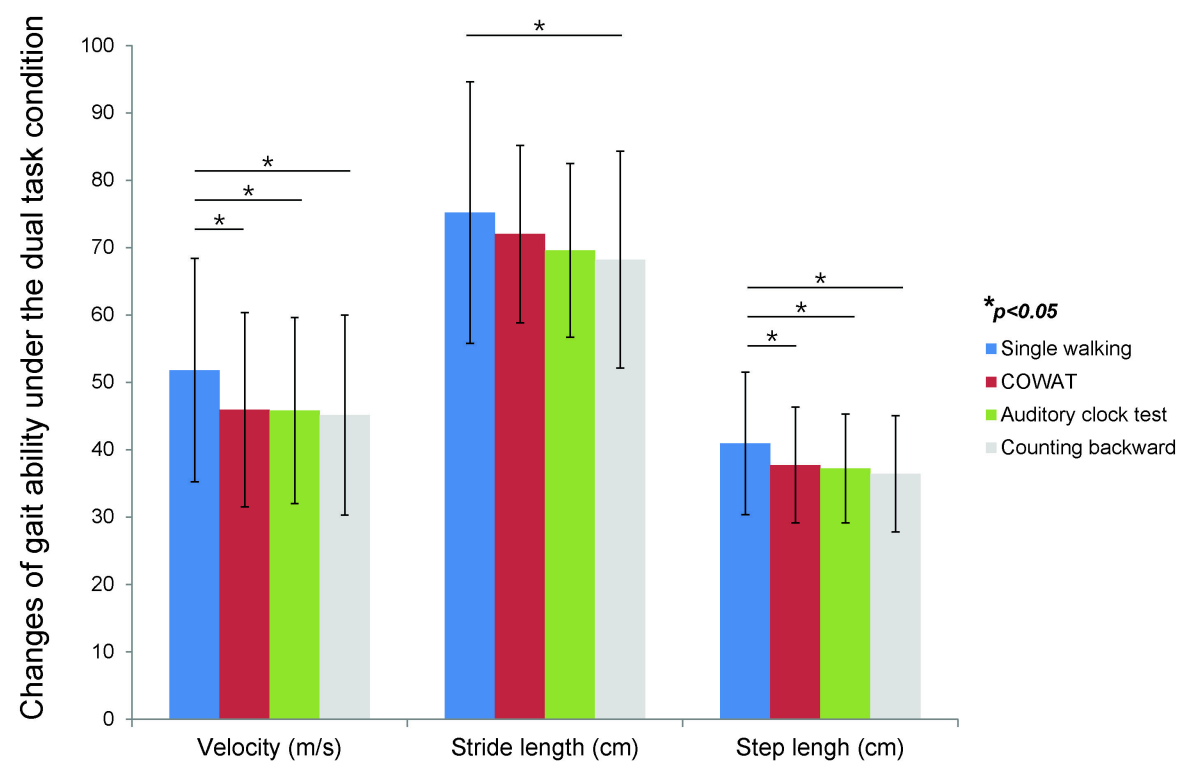

Figure 1. Changes of gait ability under the dual task conditions. COWAT: controlled oral word association test. walking ability in stroke rehabilitation. However, there is insufficient research that has investigated the change of walking ability according to the type and difficulty level of the cognitive task component of the dual task. Therefore, in this study, three different types of cognitive tasks were performed simultaneously during gait by chronic stroke survivors, and changes in walking ability according to the type and level of difficulty of the cognitive tasks were investigated. In this study, the COWAT, auditory clock test, and counting backwards were used as cognitive tasks in order to analyze the decreased walking ability with dual task performance. The results showed that velocity, stride length, and step length were significantly decreased, and that walking ability was especially remarkable when counting backwards ( $p<0.05$; Table 2 and Figure 1).

It is important to analyze the relationship between cognitive function and motor function in order to understand the recovery of motor control ability after neurological injury, such as stroke. Most daily activities require an exercise and cognitive component occurring simultaneously in order to perform activities that may be diverse and complex [21]. Prior to producing a voluntary movement, it is essential to have involuntary postural control in order to carry out multiple tasks at the same time [22]. Since involuntary postural control is organized through motor learning through cognitive, associative, and automatic stages [23], the general population does not require to have concentration on postural control in performing every day activities or movement. However, elderly with impaired bodily and cognitive func- tion, or persons with neurological damage, such as a stroke, require more concentration for daily life or to perform exercises due to decreased auto-generation of postural control [24]. In particular, persons with stroke attempt to control their posture from a previous learned form and since they lack the auto-generation of postural control, they are limited in ability to perform dual tasks [25]. According to previous studies, counting backwards is the most widely used cognitive task in dual-task evaluation and has a high correlation with decreased walking ability [26-28]. According to the volumetric distribution model, concentration is distributed according to the degree of difficulty of the task, and it is reported that tasks with greater difficulty requires higher levels of concentration $[29,30]$. According to this study, the difference in the reduction of walking ability is dependent on the level of difficulty of the cognitive tasks performed. In a previous study, it was reported that the difficulty level of the additional task applied during dual-task gait training could be an important factor in determining the training effect for stroke survivors [21]. Therefore, it is important to take into consideration the physical and cognitive functional levels when developing a dual-task training program to improve gait ability for persons affected by stroke.

First, it is difficult to generalize the results because of the small number of subjects analyzed gait variables. In the future, it is necessary to analyze various spatio-temporal variables of gait for a large number of subjects. Secondly, because of the various cognitive tasks used in the research on dual-task, it is necessary to study the dual task interference on 
various cognitive tasks.

This study investigated changes in walking ability in persons with chronic stroke according to the type and level of difficulty of the cognitive task that was added to a dual-task training program. According to the results of the study, there was a decrease in walking ability due to the addition of a cognitive task, and walking ability was especially impaired with the task of counting backwards. Therefore, it is necessary to consider the type and difficulty level of the cognitive task that is to be added in the development of a dual-task training program for stroke rehabilitation in the future, and it is necessary to investigate the dual-task interference of various cognitive tasks.

\section{Acknowledgements}

This study was supported by a grant from the Korea National Rehabilitation Center (No. 13-B-01).

\section{Conflict of Interest}

The authors declared no potential conflicts of interest with respect to the authorship and/or publication of this article.

\section{References}

1. Roerdink M, Lamoth CJ, Kwakkel G, van Wieringen PC, Beek PJ. Gait coordination after stroke: benefits of acoustically paced treadmill walking. Phys Ther 2007;87:1009-22.

2. Cho KH, Lee WH. Changes of spatio-temporal gait parameters according to experience falls in post-stroke patients. Phys Ther Rehabil Sci 2012;1:22-7.

3. Wandel A, Jørgensen HS, Nakayama H, Raaschou HO, Olsen TS. Prediction of walking function in stroke patients with initial lower extremity paralysis: the Copenhagen Stroke Study. Arch Phys Med Rehabil 2000;81:736-8.

4. Dickstein R. Rehabilitation of gait speed after stroke: a critical review of intervention approaches. Neurorehabil Neural Repair 2008;22:649-60.

5. Patla AE, Shumway-Cook A. Dimensions of mobility: defining the complexity and difficulty associated with community mobility. J Aging Phys Act 1999;7:7-19.

6. Hunter MC, Hoffman MA. Postural control: visual and cognitive manipulations. Gait Posture 2001;13:41-8.

7. Silsupadol P, Siu KC, Shumway-Cook A, Woollacott MH. Training of balance under single- and dual-task conditions in older adults with balance impairment. Phys Ther 2006;86:269-81.

8. Plummer-D'Amato P, Altmann LJ, Saracino D, Fox E, Behrman AL, Marsiske M. Interactions between cognitive tasks and gait after stroke: a dual task study. Gait Posture 2008;27:683-8.
9. Hyndman D, Pickering RM, Ashburn A. Reduced sway during dual task balance performance among people with stroke at 6 and 12 months after discharge from hospital. Neurorehabil Neural Repair 2009;23:847-54.

10. Erickson KI, Colcombe SJ, Wadhwa R, Bherer L, Peterson MS, Scalf PE, et al. Training-induced functional activation changes in dual-task processing: an FMRI study. Cereb Cortex 2007;17: 192-204.

11. Wang XQ, Pi YL, Chen BL, Chen PJ, Liu Y, Wang R, et al. Cognitive motor interference for gait and balance in stroke: a systematic review and meta-analysis. Eur J Neurol 2015;22:555e37.

12. Hyndman D, Ashburn A, Yardley L, Stack E. Interference between balance, gait and cognitive task performance among people with stroke living in the community. Disabil Rehabil 2006; 28:849-56.

13. Lacour M, Bernard-Demanze L, Dumitrescu M. Posture control, aging, and attention resources: models and posture-analysis methods. Neurophysiol Clin 2008;38:411-21.

14. Haggard P, Cockburn J. Concurrent performance of cognitive and motor tasks in neurological rehabilitation. Neuropsychol Rehabil 1998;8:155-70.

15. Cockburn J, Haggard P, Cock J, Fordham C. Changing patterns of cognitive-motor interference (CMI) over time during recovery from stroke. Clin Rehabil 2003;17:167-73.

16. Brauer SG, Morris ME. Can people with Parkinson's disease improve dual tasking when walking? Gait Posture 2010;31:229-33.

17. Dennis A, Dawes H, Elsworth C, Collett J, Howells K, Wade DT, et al. Fast walking under cognitive-motor interference conditions in chronic stroke. Brain Res 2009;1287:104-10.

18. Parker TM, Osternig LR, Lee HJ, van Donkelaar P, Chou LS. The effect of divided attention on gait stability following concussion. Clin Biomech (Bristol, Avon) 2005;20:389-95.

19. Yogev G, Giladi N, Peretz C, Springer S, Simon ES, Hausdorff JM. Dual tasking, gait rhythmicity, and Parkinson's disease: which aspects of gait are attention demanding? Eur J Neurosci 2005;22:1248-56.

20. Balasubramaniam R, Wing AM. The dynamics of standing balance. Trends Cogn Sci 2002;6:531-6.

21. Yang YR, Chen YC, Lee CS, Cheng SJ, Wang RY. Dual-task-related gait changes in individuals with stroke. Gait Posture 2007; 25:185-90.

22. Walker C, Brouwer BJ, Culham EG. Use of visual feedback in retraining balance following acute stroke. Phys Ther 2000;80: 886-95.

23. Shumway-Cook A, Woollacott MH. Motor control: theory and practical applications. 2nd ed. Philadelphia: Lippincott Williams \& Wilkins; 2001.

24. Doumas M, Rapp MA, Krampe RT. Working memory and postural control: adult age differences in potential for improvement, task priority, and dual tasking. J Gerontol B Psychol Sci Soc Sci 2009;64:193-201.

25. Brauer SG, Woollacott M, Shumway-Cook A. The influence of a concurrent cognitive task on the compensatory stepping response to a perturbation in balance-impaired and healthy elders. Gait Posture 2002;15:83-93.

26. Allali G, Assal F, Kressig RW, Dubost V, Herrmann FR, Beauchet O. Impact of impaired executive function on gait 
stability. Dement Geriatr Cogn Disord 2008;26:364-9.

27. Allali G, Kressig RW, Assal F, Herrmann FR, Dubost V, Beauchet $\mathrm{O}$. Changes in gait while backward counting in demented older adults with frontal lobe dysfunction. Gait Posture 2007;26:572-6.

28. Cho KH, Lee HJ, Lee WH. Test-retest reliability of the GAITRite walkway system for the spatio-temporal gait parameters while dual-tasking in post-stroke patients. Disabil Rehabil 2015;37: 512-6.

29. Broadbent DE. Task combination and selective intake of information. Acta Psychol (Amst) 1982;50:253-90.

30. Pashler H. Dual-task interference in simple tasks: data and theory. Psychol Bull 1994;116:220-44. 